\title{
Delayed, Stable Presentation of Complete Uterine Rupture
}

\section{Melody Rasouli*, Alyssa Stiff, McKenna Geary and Samet Albayrak}

Department of Obstetrics and Gynecology, University of Nevada, Las Vegas, USA

*Corresponding author: Melody Rasouli, Department of Obstetrics and Gynecology, University of Nevada, Las Vegas, 3016 W. Charleston Blvd., Suite 205, NV 89102, USA

\begin{abstract}
Background: Complete uterine rupture (UR) is a rare, catastrophic event.

Case: We present a rare case of fetal demise with delayed presentation of complete UR in a hemodynamically stable, asymptomatic 32-year-old woman with a history of one low transverse cesarean section and two subsequent uncomplicated vaginal births after cesarean delivery (VBAC).

Conclusion: Complete prelabor ruptures in women with a previous uterine scar are rare but are associated with catastrophic outcomes. Intrauterine fetal demise should be recognized as a sign, not necessarily a sole diagnosis, until other etiologies are excluded.
\end{abstract}

\section{Keywords}

Uterine rupture, Vaginal birth after cesarean section, Trial of labor after cesarean section

\section{Introduction}

Uterine rupture (UR) is associated with catastrophic outcomes including fetal and maternal death. UR can be classified as complete, with separation of all layers of the uterine wall, compared to incomplete, where the uterine muscle is separated and the visceral peritoneum is still intact [1]. The aim of this case is to discuss the possibility of a stable clinical patient with complete UR resulting in complete expulsion of the fetus into the abdomen with fetal demise in the late preterm period.

\section{Case}

A 32-year-old G4P3003 female with no prenatal care presented to labor and delivery triage at 36 weeks per her stated EDC with absent fetal movement for one week and no fetal heart rate determine by non- medical ultrasound center. She denied abdominal pain or vaginal bleeding; however, she recalled that she had an episode of abdominal cramping and leakage of fluid one week prior, and stopped feeling fetal movement after this date.

Her pregnancy was complicated by hypothyroidism, for which she was on levothyroxine, and reported elevated blood pressures with at home monitoring. She had one prior cesarean delivery (CD) for breech presentation and two subsequent vaginal births after cesarean (VBAC) without complications.

On initial evaluation, the patient was afebrile with a heart rate of 140 and blood pressure of 146/100. Pain score was 0 . Fetal heart tones were absent. Her abdomen was soft, non-tender without peritoneal signs. Speculum exam revealed a multiparous external cervical os without vaginal bleeding. Upon initial bedside US evaluation revealed no fetal heart rate with no intrauterine products. No intraabdominal free fluid was observed. Fetus and placenta was identified in transverse position in the upper abdomen above the uterus. Lower uterine segment appeared to be not intact. Formal ultrasound demonstrated an empty uterus with separate distorted fetal anatomy with absent cardiac activity (Figure 1). After consultation with the radiologist, an emergent magnetic resonance imaging (MRI) abdomen/pelvis was done to evaluate for UR versus uterine anomaly. On MRI, a $6 \times 6 \mathrm{~cm}$ low anterior uterine defect was identified with the fetus protruding into the abdominal cavity (Figure 2).

During laparotomy, the fetal head was encountered upon entry into the abdomen, and demised fetus was delivered. The placenta was $90 \%$ in the abdomen, and

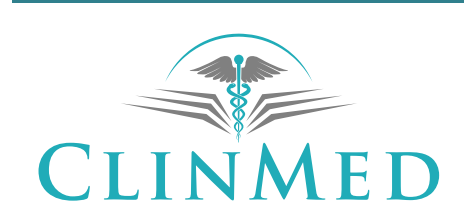

INTERNATIONAL LIBRARY 




Figure 1: Transvaginal ultrasound with empty uterus with $4 \mathrm{~mm}$ endometrial stripe and no free fluid in pelvis.

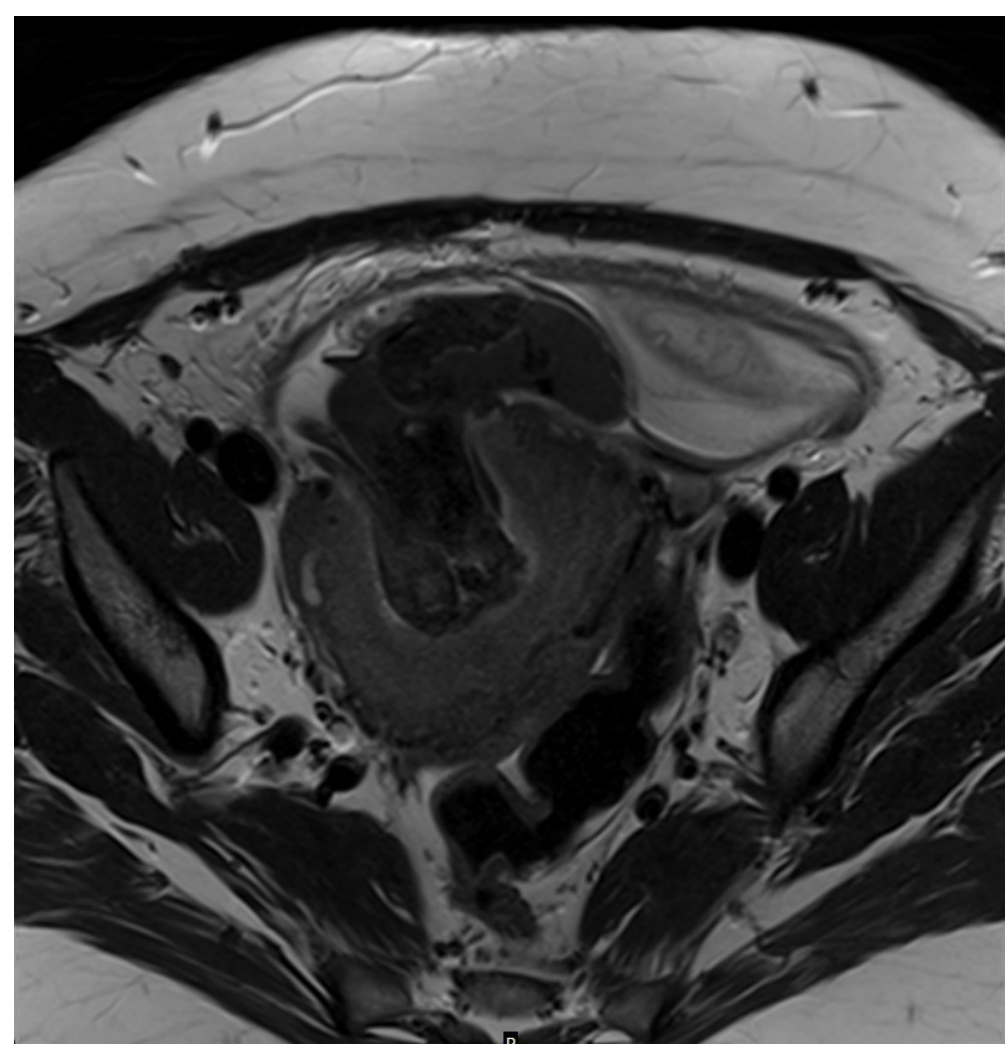

Figure 2: MRI findings. Transversal image revealing the defect in the uterine wall. Note the extruded placenta and amniotic membranes.

the remainder was removed manually from the lower uterine segment. There was no hemoperitoneum identified. The anterior uterine wall defect was noted to have scarred edges that appeared chronically healed (Figure 3). Supracervical abdominal hysterectomy was performed as the lower aspect of the incision was adhered to the urinary bladder, and intra-operative bleeding was encountered when dissecting the broad ligament. Acute chorioamnionitis was confirmed on placental pathology. Her postoperative course was uncomplicated, and she was discharged home on postoperative day 2 . Our patient presented to the clinic 


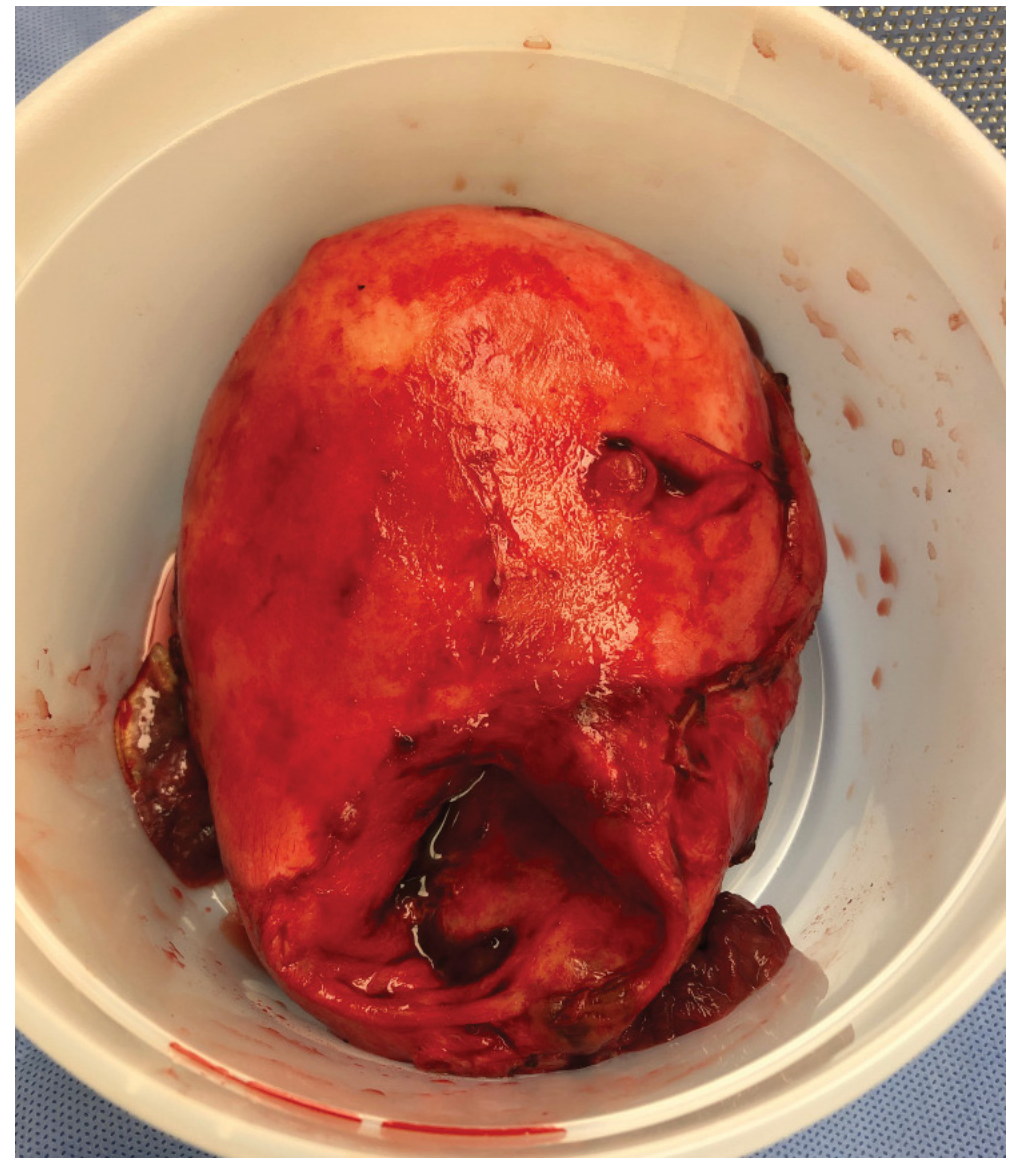

Figure 3: Cranio-caudal view of the lower uterine segment defect. Scarlike defect with non-bleeding edges.

one week later for follow-up and continued to be doing clinically well.

\section{Conclusion}

We believe this is the first case reported with subacute presentation of uterine rupture. Patient presented at an unknown time after the rupture. This case poses unique challenges for the diagnosis and management of the rupture as mentioned above. Intrauterine fetal demise should be recognized as a sign, not necessarily a sole diagnosis, until other etiologies are excluded.

Complete prelabor ruptures in women with a previous uterine scar are rare but are associated with catastrophic outcomes [1]. A retrospective populationbased study of 121,085 women identified 22 cases of prelabor complete UR (0.02\%) [2]. All 22 patients in this study presented with acute abdomen. Our patient did not have an acute abdomen or signs of hypovolemia despite complete expulsion of fetus into the abdomen and fetal demise.

Ultrasound was the first step in the evaluation of the patient and remains the first line of imaging in obstetrics. Sonographic signs of UR including a protruding portion of the amniotic sac, endometrial or myometrial defects, intraperitoneal fetal parts, extrauterine hematoma and hemoperitoneum. MRI can offer a comprehensive assessment of the uterine wall and peritoneal cavity to better identify the extent of the uterine defect, however it may delay the laparotomy.

There have been some proposed regression models to predict the chance of success for VBAC. However, there is little evidence for how to predict UR. Bujold proposes using sonographic measurements to predict the risk of UR [3]. In a study of 236 women, a uterine wall full-thickness measuring less than $2.3 \mathrm{~mm}$ at 35 to 38 weeks was significantly correlated with increased risk of rupture. Our patient did not have prenatal care, though she may have benefited from this monitoring. Routinely monitoring uterine wall thickness in patients with previous cesarean deliveries may also lead to unnecessary interventions and preterm births.

There is no standard for surgical management of complete UR. The patient's hemodynamic stability, the size and location of the uterine opening, the patient's future desire for fertility and intraoperative findings. Uterine repair, uterine repair with bilateral tubal ligation, sub-total hysterectomy or total hysterectomy are options for surgical management.

\section{Author Contributions}

Conceptualization: M.R , A.S., M.G. and S.A; Methodology: M.R, A.S. and SA; Writing-Original draft preparation: M.R., A.S. and S.A; Writing-Review and Editing: M.G. 
All authors have read and agreed to the published version of the manuscript.

\section{Funding}

This research received no external funding.

\section{Institutional Review Board Statement}

Not applicable.

\section{Informed Consent Statement}

Written informed consent has been obtained from the patient to publish this paper.

\section{Acknowledgments}

None.

\section{Conflicts of Interest}

The authors declare no conflict of interest.

\section{References}

1. Al-Zirqi I, Vangen S (2020) Prelabour uterine rupture: Characteristics and outcomes. BJOG 127: 1637-1644.

2. American College of Obstetricians and Gynecologists (2019) Vaginal birth after cesarean delivery: Practice Bulletin Number 205. Obstet Gynecol 133: e110-127.

3. Bujold E, Jastrow N, Simoneau J, Brunet S, Gauthier RJ (2009) Prediction of complete uterine rupture by sonographic evaluation of the lower uterine segment. Am J Obstet Gynecol 201: 320.e1-320.e6. 